\title{
Scattering of Classical and Quantum Particles by Impulsive Fields
}

\author{
Herbert Balasin* Peter C. Aichelburg ${ }^{\dagger}$ \\ Institut für $\quad$ Fakultät für Physik \\ Theoretische Physik Gravitationsphysik \\ TU-Wien Universität Wien
}

March 22, 2022

\begin{abstract}
We investigate the scattering of classical and quantum particles in impulsive backgrounds fields. These fields model short outbursts of radiation propagating with the speed of light. The singular nature of the problem will be accounted for by the use of Colombeau's generalized function which however give rise to ambiguities. It is the aim of the paper to show that these ambiguities can be overcome by implementing additional physical conditions, which in the non-singular case would be satisfied automatically. As example we discuss the scattering of classical, Klein-Gordon and Dirac particles in impulsive electromagnetic fields.
\end{abstract}

*hbalasin@tph.tuwien.ac.at

†aichelp8@univie.ac.at 


\section{Introduction}

In the following we consider the scattering of classical (point-like) as well as quantum (waves) particles by impulsive background fields. That is to say, the particles interact with a field that is solely concentrated on a null hyperplane described by a delta-like singularity. The physical motivation is to model the behavior of particles affected by extreme short outbursts of radiation such as observed in supernovae explosions, gamma-ray bursts or the fields of ultra-short laser pulses produced in the laboratory. Since, from the spacetime point of view, the particles move freely "above" and "below" the pulse-hyperplane the solution of the equations of motion is turned into a matching problem for free solutions.

The mathematical price for this simplified physical description comes in the form of non-linear operations performed on distributional objects. The adequate framework is provided by the algebra of new generalized functions $\mathcal{G}$ of Colombeau [1]. It circumvents the Schwarz impossibility result, that claims the non-existence of a (differential) algebra extending the continuous functions and containing the distributions, by requiring only the $C^{\infty}$-functions to be a sub-algebra.

Early work by DeVega and Sanchez [2] and Lousto and Sanchez [3] discuss the scattering of Klein-Gordon and Dirac fields in a special class of impulsive gravitational backgrounds. These geometries can be obtained from the ultrarelativistic limit of black hole space-times (AS-geometries [4] and generalizations thereof 1 ). The authors notice a particular regularization dependence of their result.

On the other hand Kunzinger and Steinbauer [6] have investigated the behavior of geodesics in general impulsive pp-wave backgrounds via rigorously embedding the equations into the Colombeau algebra. They show that a (unique) solutions to the geodesic as well as the geodesic deviation equation exist in $\mathcal{G}$ which possess a reasonable macroscopic, that is distributional, "shadow". These results are in accordance with earlier work by Balasin [7].

In the present paper we follow a similar strategy as in [7] whence extended to the field context. Distributional equality will be replaced by association which is the corresponding notion in $\mathcal{G}$. We find that in spite of the singular character the nonlinear operations yield associated distributional objects containing, however, finite undetermined quantities, which is to be expected

\footnotetext{
${ }^{1}$ For an initial value approch to impulsive gravitational waves cf. [5]
} 
[1. In order to define their values from a physical point of view we make use of conservation laws. These would follow in the smooth context via nonlinear operations which, in general however, break association. We believe that this method provides a systematic way to decide upon the "regularization dependence" in [2] without relying on the heavy machinery used in [6] (in fact being closer to the approach used in [1]) In sec. 1 we briefly recall the definition of the Colombeau algebra and discuss the properties of association. As a warm up, we start in sec. 2 by considering a particle subject to a potential acting only at a single instant of time. Already in the Newtonian (classical) case, care must be taken in handling the non-linearities coming from the potential, but the solution is uniquely determined. In contrast, the corresponding Schrödinger problem, in sec. 3, leads to an undetermined quantity. The reason is the association of the product of the $\delta$ term in the potential with the $\theta$ functions of the matched free solutions. Rather than stipulating its value by hand, we require the conservation of probability through the pulse, which does not follow from the equation since it involves non-linear operations which in general break distributional equality, i.e. association. From this we find via a Cayley-transform the uniquely defined transition amplitude. In a next step, in sec. 4, we consider a classical particle in an electromagnetic pulse and the corresponding Klein-Gordon equation as its quantum version (sec. 5). Although the situation is more involved the strategy is precisely the same as in the Newton-Schrödinger case. Here already the classical problem gives rise to an ambiguity which can be fixed by requiring that the length of the tangent vector to be preserved.

The case of the Klein-Gordon field is still more involved because it leads to two undetermind constants, which at first sight seems hopeless for obtaining a unique solution. However, we show that by the physical requirement that the Klein-Gordon current is conserved across the pulse, the two constants are determined and thus the matching is unique. Finally we focus on relativistic particles with spin i.e. we consider the impact of an electromagnetic pulse on Dirac-particles. Again undefined quantities (constants) arise. It is natural to impose the conservation of the Dirac current across the pulse which then gives the unique transition amplitude. 


\section{The method}

The Colombeau algebra $\mathcal{G}$ consists of one-parameter families of $C^{\infty}$ functions, $\left(f_{\epsilon}(x)\right)_{(0<\epsilon<1)}$ subject to certain growth-conditions in $\epsilon$. Its elements may be thought of as being regularizations of distributional (and even more singular) objects. Distributions form a linear subspace of $\mathcal{G}$. This subspace is not canonical in the same sense as $S U(2)$ is not a canonical subgroup of $S L(2, \mathbb{C})$. In particular this means that there are many different " $\delta$-functions" in $\mathcal{G}$. This property is reflected by an equivalence relation on the algebra called association and denoted by $\approx$

$$
\left(f_{\epsilon}(x)\right) \approx\left(g_{\epsilon}(x)\right) \quad \text { iff } \quad \lim _{\epsilon \rightarrow 0} \int d^{n} x\left(f_{\epsilon}-g_{\epsilon}\right)(x) \varphi(x)=0 \quad \forall \varphi \in C_{0}^{\infty}\left(\mathbb{R}^{n}\right)
$$

Objects in the same equivalence class may differ in their micro-aspect. That is to say although they are in general different objects in $\mathcal{G}$, they all correspond (if it exists) to the same distribution. In this regard we may think of association as a kind of coarse-graining of $\mathcal{G}$. Distributionally well-defined, i.e. linear, operations have well-defined analogs in $\mathcal{G}$, which are compatible with their macro-aspect, meaning they do not break association

$$
\left(f_{\epsilon}\right) \approx\left(g_{\epsilon}\right) \quad\left\{\begin{array}{l}
\Rightarrow f \cdot\left(f_{\epsilon}\right) \approx f \cdot\left(g_{\epsilon}\right) \\
\Rightarrow\left(\partial_{\alpha} f_{\epsilon}\right) \approx\left(\partial_{\alpha} g_{\epsilon}\right)
\end{array}\right.
$$

On the other hand, non-linear operations on different representatives of an association-class do in general break association. This means that upon nonlinear operations different micro-aspects may get magnified to the macrolevel. A simple, nevertheless important, example is given by

$$
\theta \cdot \delta \approx A \delta
$$

where $A$ denotes a constant. This simply states that $A$ is the result of the relative micro-aspects of the two elements of $\mathcal{G}$ that are associated to $\theta$ and $\delta$ respectively.

As a special case we have

$$
\left(\theta^{2}\right)^{\prime} \approx \theta \cdot \theta^{\prime} \approx \frac{1}{2} \delta
$$

or more generally by

$$
\theta^{n} \theta^{\prime} \approx \frac{1}{n+1} \delta
$$


These results follow from $\theta^{n} \approx \theta$ and the compatibility of association with differentiation. In both the above cases the constant is determined regardless of the representative $\theta$. This reflects the fact that the relative micro-aspect between $\theta$ and $\theta^{\prime}$ and $\theta^{n}$ and $\theta^{\prime}$ respectively is independent of the representative. Notice that all $\theta^{n}$ are again $\theta$-functions, i.e. are associated to the $\theta$-distribution. However, their different micro-aspects relative to $\theta^{\prime}$ a representative of $\delta$ gets magnified to the macro-level.

\section{Newtonian particle}

Having prepared the stage, let us apply the formalism to the simple-most classical system: a particle under the influence of a potential acting only at an instant of time, described by the Newtoniam equation of motion.

$$
m \ddot{x}^{i}(t)+\delta(t) \partial_{i} V\left(x^{m}(t)\right) \approx 0 .
$$

We have chosen weak equality because the $\delta$ term representing the idealized action of the force during the shortest possible period of time. Since the force acts only at $t=0$ the trajectory is given by

$$
\begin{gathered}
x^{i}(t)=\theta_{+}(t) x_{+}^{i}(t)+\theta_{-}(t) x_{-}^{i}(t), \quad x_{+}^{i}(t), x_{-}^{i}(t) \in C^{\infty}(\mathbb{R}) \\
\theta_{+}(t)+\theta_{-}(t)=1,
\end{gathered}
$$

We require $x_{-}^{i}(t)$ and $x_{+}^{i}(t)$ to be solutions of the free equations of motions before and after the pulse respectively.

Upon insertion into (6) gives

$$
\begin{aligned}
& m\left(x_{+}^{i}(0)-x_{-}^{i}(0)\right) \delta^{\prime}(t)+m\left(\dot{x}_{+}^{i}(0)-\dot{x}_{-}^{i}(0)\right) \delta(t)+ \\
& \theta_{+}(t) m \ddot{x}_{+}^{i}(t)+\theta_{-}(t) m \ddot{x}_{-}^{i}(t)+\delta(t) \partial_{i} V\left(x^{m}(t)\right) \approx 0 .
\end{aligned}
$$

The last term contains, via the association process, undetermined constants as pre-factors of $\delta(t)$, i.e. $\delta(t) \partial_{i} V\left(x^{m}(t)\right) \approx C_{i} \delta(t)$.

In a first step, multiplication with the $C^{\infty}$ function $t$ ensures the vanishing of all the terms in (6) except the $\delta^{\prime}$ term, since $t \delta^{\prime}$ is not $\approx 0$ tells us that the coefficient has to vanish, i.e. $x_{+}^{i}(0)=x_{-}^{i}(0)$. This then in turn determines the prefactor to be $C_{i}=\partial_{i} V\left(x^{m}(0)\right)$.

So we are only left with the $\delta$ function coefficient

$$
m \dot{x}_{+}^{i}(0)-m \dot{x}_{-}^{i}(0)=-\partial_{i} V\left(x^{m}(0)\right),
$$


where $x^{i}(0)$ has now a well-defined meaning. The junction condition has a simple physical interpretation as mapping the $t=0$ conditions of for $x_{-}$onto the $t=0$ condition for $x_{+}$, i.e.

$$
\left(x_{-}^{i}(0), \dot{x}_{-}^{i}(0)\right) \mapsto\left(x_{+}^{i}(0), \dot{x}_{+}^{i}(0)\right)=\left(x_{-}^{i}(0), \dot{x}_{-}^{i}(0)-\frac{1}{m} \partial_{i} V\left(x^{m}(0)\right)\right) .
$$

As expected, the freely moving particle gets a kick at $t=0$, thereby changing its velocity according to the applied force.

\section{$3 \quad$ Schrödinger particle}

Quantization of the above system is described by the corresponding timedependent, Schrödinger equation

$$
i \dot{\psi}(x, t)+\left(-\frac{\partial^{2}}{2 m}+\delta(t) V(x)\right) \psi(x, t) \approx 0
$$

Proceeding in the same way as with the classical system we combine two solutions before and after $t=0$, i.e.

$$
\psi(x, t)=\theta_{+}(t) \psi_{+}(x, t)+\theta_{-}(t) \psi_{-}(x, t) .
$$

where we assume that $\psi_{-}(x, t)$ and $\psi_{+}(x, t)$ satisfy the free Schrödinger equation.

Inserting (91) into (8) we are left with

$$
i \delta(t)\left(\psi_{+}(x, 0)-\psi_{-}(x, 0)\right) \approx \delta(t) V(x)\left(A \psi_{+}(x, 0)+(1-A) \psi_{-}(x, 0)\right) .
$$

The above relation made implicit use of $\theta \cdot \delta \approx A \delta$, which expresses our ignorance about the microscopic relation between $\theta$ and $\delta$. As in the classical regime this relates the data $\psi_{-}(x, 0)$ before the shock to the data $\psi_{+}(x, 0)$ after the shock

$$
\psi_{+}(x, 0)=\frac{1-i(1-A) V(x)}{1+i A V(x)} \psi_{-}(x, 0) .
$$

There is however an important difference, that manifests itself in the appearance of the arbitrary constant $A$. It signals that we have oversimplified the physical description of the system by making it too singular. Following [1] the mathematical description would need further specification. It is precisely 
additional physical input that allows to determine $A$. For general smooth solutions of the Schrödinger equation we have conservation of the probability current. However due to the weak nature of our equation current conservation is no longer one of its consequences. In order to preserve the physical interpretation of the Schrödinger equation we have

$$
\begin{aligned}
\dot{\rho}+\partial_{i} j^{i} \approx 0, & \rho=\bar{\psi} \psi, \quad j^{i}=\frac{1}{2 m i}\left(\bar{\psi} \partial_{i} \psi-\psi \partial_{i} \bar{\psi}\right), \\
\rho & \approx \theta_{+} \bar{\psi}_{+} \psi_{+}+\theta_{-} \bar{\psi}_{-} \psi_{-}, \\
j^{i} & \approx \frac{1}{2 m i}\left(\theta_{+}\left(\bar{\psi}_{+} \partial_{i} \psi_{+}-\psi_{+} \partial_{i} \bar{\psi}_{+}\right)+\theta_{-}\left(\bar{\psi}_{-} \partial_{i} \psi_{-}-\psi_{-} \partial_{i} \bar{\psi}_{-}\right)\right), \\
\dot{\rho}+\partial_{i} j^{i} & \approx \delta\left(\bar{\psi}_{+} \psi_{+}-\bar{\psi}_{-} \psi_{-}\right) .
\end{aligned}
$$

Using (10) has the immediate consequence

$$
\bar{\psi}_{+} \psi_{+}=\left|\frac{1-i(1-A) V}{1+i A V}\right|^{2} \bar{\psi}_{-} \psi_{-} .
$$

Therefore probability-current conservation is only achieved if the pre-factor in the last equation has unit length, thereby fixing $A$ to be $1 / 2$. With this, the data below the pulse are mapped uniquely via a Cayley-transform of the reduced potential into the data above the pulse.

$$
\psi_{+}(x, 0)=\frac{1-\frac{i}{2} V(x)}{1+\frac{i}{2} V(x)} \psi_{-}(x, 0) .
$$

We mention that our formalism also works in the more common situation of a spatially "impulsive" potential $V\left(x^{m}\right)=\delta(n x) \tilde{V}\left(\tilde{x}^{m}\right)$ where similar ambiguities arise, but do not contribute to the result as for the Newtonian particle.

\section{Lorentz particle}

Turning to a relativistic setting it is natural to consider disturbances that travel with the fundamental velocity i.e. along null, rather than on $t=$ const surfaces. Therefore, we look at the scattering by impulsive electromagnetic fields which are completely concentrated on a null hyperplane. The vector potential $A_{a}$ and the field-strength $F_{a b}$ take the form

$$
A_{a}=f\left(p x, \tilde{x}^{m}\right) p_{a}, F_{a b}=2 \tilde{\partial}_{[a} f p_{b]}, \quad f\left(p x, \tilde{x}^{m}\right)=\delta(p x) \tilde{f}\left(\tilde{x}^{m}\right)
$$


where $p^{a}$ denotes (a covariantly constant) null vector-field and $\tilde{x}^{i}$ denotes the spacelike coordinates of the two-dimensional subspace orthogonal to $p^{a}$ and a conjugate null direction $\bar{p}^{a}$. Here we follow the coordinate free notation of Penrose [8]. However, if one introduces the coordinates $\left(u, v, \tilde{x}^{m}\right)$ in Minkowski space

$$
d s^{2}=2 d u d v-d \tilde{x}^{m} d \tilde{x}^{m}
$$

and chosing $p=\partial_{v}$ and $\bar{p}=\partial_{u}$, then $p x=u$ and $\bar{p} x=v$.

$F_{a b}$ satisfies the vacuum equations provided

$$
\Delta_{2} \tilde{f}\left(\tilde{x}^{m}\right)=0
$$

The motion of test-particles $x^{a}(s)$ is described by the Lorentz-force law

$$
\begin{aligned}
m \ddot{x}^{a}+e F^{a}{ }_{b} \dot{x}^{b} & =0 \\
m \ddot{x}^{a}+e\left((p \dot{x}) \tilde{\partial}^{a} f-(\dot{\tilde{x}} \tilde{\partial}) f p^{a}\right) & =0
\end{aligned}
$$

which becomes upon decomposition with respect to $p^{a}, \bar{p}^{a}$ and their orthogonal complement

$$
\begin{aligned}
p \ddot{x} & =0, \\
\bar{p} \ddot{x}+\frac{e}{m}(\dot{\tilde{x}} \tilde{\partial}) f & =0, \\
\ddot{\tilde{x}}+\frac{e}{m}(p \dot{x}) \tilde{\partial} f & =0,
\end{aligned}
$$

where we have suppressed the indices in the two-dimensional (tilde) part and made use of the normalization $p \cdot \bar{p}=1$. The first equation of (14) tells us that $p x$ may be chosen as an "affine" parameter for the trajectory $\left((p x)^{*}=\alpha\right)$ unless we consider motion within a hyperplane orthogonal to $p^{a}$. Taking into account the impulsive nature of the profile $f$ the equation will be considered as weak equality within the Colombeau algebra 2

$$
\begin{aligned}
(\bar{p} x)^{\prime \prime}(p x)+\frac{e}{\alpha m} \delta(p x)\left(\tilde{x}^{i \prime}(p x) \tilde{\partial}_{i} \tilde{f}\left(\tilde{x}^{m}(p x)\right)\right. & \approx 0 \\
\tilde{x}^{i \prime \prime}(p x)+\frac{e}{\alpha m} \delta(p x) \tilde{\partial}_{i} \tilde{f}\left(\tilde{x}^{m}(p x)\right) & \approx 0 .
\end{aligned}
$$

\footnotetext{
${ }^{2}$ Here and in the following notation like $(\bar{p} x)(p x) \equiv(\bar{p} x)(u)$ denotes the dependence of $\bar{p} x$ and similar expressions on the affine parameter $p x=u$
} 
Since the electromagnetic field is completely concentrated on the plane $p x=0$ the particle moves freely "above" and "below" the pulse, i.e.

$$
\begin{aligned}
(\bar{p} x)^{\prime \prime}(p x) & =\theta_{+}(p x)\left(\bar{p} x_{+}\right)(p x)+\theta_{-}(p x)\left(\bar{p} x_{-}\right)(p x), \\
\tilde{x}^{i \prime \prime}(p x) & =\theta_{+}(p x) \tilde{x}_{+}^{i}(p x)+\theta_{-}(p x) \tilde{x}_{-}^{i}(p x) .
\end{aligned}
$$

The second equation of (15) is identical to that for the Newtonian particle. Therefore the junction conditions become

$$
\begin{aligned}
& \tilde{x}^{i}{ }_{+}(0)=\tilde{x}^{i}{ }_{-}(0), \\
& \tilde{x}^{i}{ }_{+}(0)=\tilde{x}^{i}{ }_{-}(0)-\frac{e}{\alpha m} \tilde{\partial}_{i} \tilde{f}\left(\tilde{x}^{m}(0)\right) .
\end{aligned}
$$

Let us now take a closer look a the first equation of (15)).

$$
\begin{gathered}
\left(\left(\bar{p} x_{+}\right)(0)-\left(\bar{p} x_{-}\right)(0)\right) \delta^{\prime}(p x)+\left(\left(\bar{p} x_{+}\right)^{\prime}(0)-\left(\bar{p} x_{-}\right)^{\prime}(0)\right) \delta(p x)+ \\
\frac{e}{\alpha m} \delta(p x)\left(\theta_{+}^{\prime}(p x)\left(\tilde{x}_{+}^{i}(p x)-\tilde{x}_{-}^{i}(p x)\right)+\right. \\
\left.\theta_{+}(p x) \tilde{x}_{+}^{\prime i}(p x)+\theta_{-} \tilde{x}_{-}^{\prime i}(p x)\right) \tilde{\partial}_{i} \tilde{f}\left(\tilde{x}^{m}(p x)\right) \approx 0 .
\end{gathered}
$$

Due to the appearance of products like $\theta^{\prime}(p x) \cdot \delta(p x)$ the above expression makes only sense within the algebra. Multiplication with $p x$ and taking into account the junction conditions for $\tilde{x}^{i}(p x)$ along the lines of the Newtonian particle, shows that the coefficient of the $\delta^{\prime}(p x)$ term has to vanish separately, i.e.

$$
\left(\bar{p} x_{+}\right)(0)=\left(\bar{p} x_{-}\right)(0) .
$$

From the remaining expression we obtain

$$
\left(\bar{p} x_{+}\right)^{\prime}(0)-\left(\bar{p} x_{-}\right)^{\prime}(0)+\frac{e}{\alpha m}\left(A \tilde{x}_{+}^{\prime i}(0)+(1-A) \tilde{x}_{-}^{i}(0)\right) \tilde{\partial}_{i} \tilde{f}\left(\tilde{x}^{m}(0)\right)=0,
$$

where, as before, the (remaining) constant $A$ arises from $\theta(p x) \cdot \delta(p x) \approx$ $A \delta(p x)$. Let us pause for a moment and compare our results with the Newtonian case. Although we have obtained the arbitrary constant $A$ in very much the same way, this arbitrariness already appears at the classical level. In order to fix this constant we will invoke a consequence of the equation of motions for smooth solutions, namely the fact that the length of the tangent vector remains constant along the trajectory 


$$
\begin{aligned}
& -2(\bar{p} x)^{\prime}(p x)+\left(\tilde{x}^{\prime}(p x)\right)^{2} \approx \text { const, } \\
& -2\left(\theta_{+}(p x)\left(\bar{p} x_{+}\right)^{\prime}(p x)+\theta_{-}(p x) \bar{p} x_{-}^{\prime}(p x)\right)+\left(\theta_{+}^{\prime}(p x)\left(\tilde{x}_{+}(p x)-\tilde{x}_{-}(p x)\right)+\right. \\
& \left.\theta_{+}(p x) \tilde{x}_{+}^{\prime}(p x)+\theta_{-}(p x) \tilde{x}_{-}^{\prime}(p x)\right)^{2} \approx \text { const }, \\
& \theta_{+}(p x)\left(-2 \bar{p} x_{+}^{\prime}(p x)+\left(\tilde{x}_{+}^{\prime}(p x)\right)^{2}\right)+\theta_{-}(p x)\left(-2 \bar{p} x_{-}^{\prime}(p x)+\left(\tilde{x}_{-}^{\prime}(p x)\right)^{2}\right) \approx \text { const },
\end{aligned}
$$

Since differentiation does not break association, we have

$$
\begin{aligned}
& \left(-2 \bar{p} x_{+}^{\prime}(0)+\left(\tilde{x}_{+}^{\prime}(0)\right)^{2}+2 \bar{p} x_{-}^{\prime}(0)-\left(\tilde{x}_{-}^{\prime}(0)\right)^{2}\right) \delta(p x) \approx 0 \\
& 2 \frac{e}{\alpha m}\left(\tilde{x}_{-}^{\prime i}(0)-A \frac{e}{\alpha m} \tilde{\partial}_{i} \tilde{f}\right) \tilde{\partial}_{i} \tilde{f}+\left(\tilde{x}_{-}^{\prime}(0)-\frac{e}{\alpha m} \tilde{\partial} \tilde{f}\right)^{2}-\left(\tilde{x}_{-}^{\prime}(0)\right)^{2}=0 .
\end{aligned}
$$

This condition is only satisfied if $A$ is taken to be $1 / 2$. Thus, summing up and denoting the "jump" at $p x=0$ by [], we have:

$$
\begin{aligned}
{\left[\tilde{x}^{i}\right] } & =0 \\
{\left[\tilde{x}^{i \prime}\right] } & =-\frac{e}{\alpha m} \tilde{\partial}_{i} \tilde{f}\left(\tilde{x}^{m}(0)\right) \\
{[\bar{p} x] } & =0 \\
{\left[\bar{p} x^{\prime}\right] } & =-\frac{e}{\alpha m}\left(\tilde{x}_{-}^{\prime}(0) \tilde{\partial} \tilde{f}\left(\tilde{x}^{m}(0)\right)\right)+\left(\frac{e}{\alpha m}\right)^{2}\left(\tilde{\partial} \tilde{f}\left(\tilde{x}^{m}(0)\right)\right)^{2}
\end{aligned}
$$

\section{Klein-Gordon particle}

Quantization gives rise to

$$
\begin{aligned}
& \left(\eta^{a b} \hat{P}_{a} \hat{P}_{b}-m^{2}\right) \Phi=0 \\
& \text { where } \hat{P}_{a} \text { is given by } \hat{P}_{a}=\left(\hat{p}_{a}-e A_{a}\right) \quad \hat{p}_{a}=\frac{1}{i} \partial_{a} \\
& \left(\eta^{a b}\left(\partial_{a}-i e A_{a}\right)\left(\partial_{b}-i e A_{b}\right)+m^{2}\right) \Phi=0 .
\end{aligned}
$$

Using the specific form of the potential (11) and taking into account the lightlike nature of $p^{a}$, the above expression simplifies to

$$
\left(\partial^{2}+m^{2}-2 i e f(p \partial)\right) \Phi=0 .
$$

The standard decomposition $\Phi=\theta_{+} \Phi_{+}+\theta_{-} \Phi_{-}$, resulting from the impulsive nature of $f$, i.e. $f=\delta(p x) \tilde{f}$ yields upon insertion into (17) 


$$
\begin{aligned}
2\left((p \partial) \Phi_{+}-(p \partial) \Phi_{-}\right) \delta+ & \theta_{+}\left(\partial^{2}+m^{2}\right) \Phi_{+}+\theta_{-}\left(\partial^{2}+m^{2}\right) \Phi_{-}+ \\
- & 2 i e \delta \tilde{f}\left(A(p \partial) \Phi_{+}+(1-A)(p \partial) \Phi_{-}\right) \approx 0
\end{aligned}
$$

where in order to have well-defined products of singular quantities (17) has been promoted to a weak statement within the Colombeau algebra. Since $\Phi_{+}, \Phi_{-}$satisfy the free Klein-Gordon equation "above" and "below" the pulse respectively, we find for the mapping from the final data of $\Phi_{-}$to the initial data of $\Phi_{+}$

$$
(p \partial) \Phi_{+}-(p \partial) \Phi_{-}=i e \tilde{f}\left(A(p \partial) \Phi_{+}+(1-A)(p \partial) \Phi_{-}\right)
$$

Once again we encounter the notorious parameter $A$ resulting from $\theta \cdot \delta \approx$ $A \delta$. The (complex) Klein-Gordon equation gives rise to a conserved current $j_{a}=(1 / i)\left(\bar{\Phi} D_{a} \Phi-D_{a} \bar{\Phi} \Phi\right), D_{a} \Phi=\left(\partial_{a}-i e A_{a}\right) \Phi, D_{a} \bar{\Phi}=\left(\partial_{a}+i e A_{a}\right) \bar{\Phi}$ for smooth initial data. Since current conservation may no longer be deduced from the singular equation of motion, we will require it to hold separately.

$$
\begin{aligned}
j^{a} \approx & \frac{1}{i}\left(\left(\bar{\Phi}_{-} \Phi_{+}-\bar{\Phi}_{+} \Phi_{-}\right) p^{a} \delta-2 i e \tilde{f}\left(B \bar{\Phi}_{+} \Phi_{+}+(A-B)\left(\bar{\Phi}_{+} \Phi_{-}+\bar{\Phi}_{-} \Phi_{+}\right)+\right.\right. \\
& \left.\left.(1-2 A+B) \bar{\Phi}_{-} \Phi_{-}\right) p^{a} \delta\right)+\theta_{+} j_{+}^{a}+\theta_{-} j_{-}^{a} \\
\partial \cdot j \approx & \frac{1}{i}\left((p \partial)\left(\bar{\Phi}_{-} \Phi_{+}-\bar{\Phi}_{+} \Phi_{-}\right)+\left(\bar{\Phi}_{+}(p \partial) \Phi_{+}-(p \partial) \bar{\Phi}_{+} \Phi_{+}\right)\right. \\
& -\left(\bar{\Phi}_{-}(p \partial) \Phi_{-}-(p \partial) \bar{\Phi}_{-} \Phi_{-}\right)-2 i e \tilde{f}(p \partial)\left(B \bar{\Phi}_{+} \Phi_{+}+\right. \\
& \left.\left.+(A-B)\left(\bar{\Phi}_{+} \Phi_{-}+\bar{\Phi}_{-} \Phi_{+}\right)+(1-2 A+B) \bar{\Phi}_{-} \Phi_{-}\right)\right) \approx 0
\end{aligned}
$$

This entails

$$
\begin{aligned}
\left(( p \partial ) \left(\bar{\Phi}_{-} \Phi_{+}\right.\right. & \left.-\bar{\Phi}_{+} \Phi_{-}\right)+\left(\bar{\Phi}_{+}(p \partial) \Phi_{+}-(p \partial) \bar{\Phi}_{+} \Phi_{+}\right)- \\
& -\left(\bar{\Phi}_{-}(p \partial) \Phi_{-}-(p \partial) \bar{\Phi}_{-} \Phi_{-}\right)-2 i e \tilde{f}(p \partial)\left(B \bar{\Phi}_{+} \Phi_{+}+\right. \\
& \left.\left.+(A-B)\left(\bar{\Phi}_{+} \Phi_{-}+\bar{\Phi}_{-} \Phi_{+}\right)+(1-2 A+B) \bar{\Phi}_{-} \Phi_{-}\right)\right)=0
\end{aligned}
$$

The expression for the current $j^{a}$ contains an additional arbitrary constant $B$, which arises from the non-linear relation $\theta^{2} \cdot \delta \approx B \delta$. Its appearance seems to completely spoil our strategy to use the conservation law to fix the value of $A$, since it seems we now need another equation to determine the value of $B$. However, this conclusion is premature, since due to local nature of the 
conditon (21) we actually have an infinite number of conditions for $A$ and $B$ and thus an overdetermined system. Re-arranging the first three in (21) in the form containing expression involving the left-hand-side of (18) and its complex-conjugate, which upon inserting the corresponding right-hand-side yields after simply comparing coefficients $A=1 / 2$ and $B=1 / 4$. This is actually a non-trivial statement according to the above mentioned (infinite) over-determinacy, which shows that the (naive) guess that $\delta=\theta_{+}^{\prime}$, which would reproduce $A=1 / 2$ in all the previous cases is inconsistent with the required value for $B$. So as a bonus from current conservation the expression for the current $j^{a}$ is associated to

$$
j^{a} \approx \theta_{+} j_{+}^{a}+\theta_{-} j_{-}^{a}+\delta \bar{\Phi}_{-} \Phi_{-} \frac{e \tilde{f}}{1+\frac{e^{2}}{4} \tilde{f}^{2}} p^{a},
$$

which contains an extra piece streaming tangential to the hyperplane of the pulse along its generators. Thus we finally obtain for the junction conditions

$$
(p \partial) \Phi_{+}=\frac{1-i \frac{e}{2} \tilde{f}}{1+i \frac{e}{2} \tilde{f}}(p \partial) \Phi_{-}
$$

which once again take the form of a Cayley-transform. Note that the matching conditions are not sufficient to determine the solution uniquely. To solve the characteristic initial value problem would require additional boundary condition along a $(\bar{p} x)=v=$ const. surface.

\section{Dirac particle}

We will now consider a charged particle with spin one-half subject to an impulsive electromagnetic field. We therefore turn to Dirac's equation, which is written in two-spinor form

$$
\begin{array}{lr}
\hat{P}_{A A^{\prime}} \psi^{A}=\frac{m}{\sqrt{2}} \chi_{A^{\prime}} & \text { where } \quad \hat{P}_{A A^{\prime}}=\frac{1}{i} \nabla_{A A^{\prime}} \\
\hat{P}^{A A^{\prime}} \chi_{A^{\prime}}=\frac{m}{\sqrt{2}} \psi^{A} & \text { and } \nabla_{A A^{\prime}}=\partial_{A A^{\prime}}-i e A_{A A^{\prime}}
\end{array}
$$

For he specific form of the potential (11) this becomes

$$
\begin{aligned}
& \partial_{A A^{\prime}} \psi^{A}-i \frac{m}{\sqrt{2}} \chi_{A^{\prime}}=i e f o_{A^{\prime}} O_{A} \psi^{A} \\
& \partial^{A A^{\prime}} \chi_{A^{\prime}}-i \frac{m}{\sqrt{2}} \psi^{A}=i e f O^{A} O^{A^{\prime}} \chi_{A^{\prime}} \quad \text { where } p^{a}=p^{A A^{\prime}}=O^{A} O^{A^{\prime}}
\end{aligned}
$$


For an impulsive profile $f=\tilde{f} \delta(p x)$ and the decomposition of $\psi^{A}=\theta_{+} \psi_{+}^{A}+$ $\theta_{-} \psi_{-}^{A}$ and $\chi^{A^{\prime}}=\theta_{+} \chi_{+}^{A^{\prime}}+\theta_{-} \chi_{-}^{A^{\prime}}$ into solutions of the free equation above and below the pulse respectively, we find

$$
\begin{aligned}
& \delta(p x)\left(o_{A^{\prime}}\left(o_{A} \psi_{+}^{A}-o_{A} \psi_{-}^{A}\right)-i e \tilde{f}\left(A o_{A} \psi_{+}^{A}+(1-A) o_{A} \psi_{-}^{A}\right)\right) \approx 0 \\
& \delta(p x)\left(o^{A}\left(o^{A^{\prime}} \chi_{A^{\prime}+}-o^{A^{\prime}} \chi_{A^{\prime}-}\right)-i e \tilde{f}\left(A o^{A^{\prime}} \chi_{A^{\prime}+}+(1-A) o^{A^{\prime}} \chi_{A^{\prime}-}\right)\right) \approx 0
\end{aligned}
$$

which in turn yields

$$
\begin{aligned}
& o_{A} \psi_{+}^{A}-o_{A} \psi_{-}^{A}=-i e \tilde{f}\left(A o_{A} \psi_{+}^{A}+(1-A) o_{A} \psi_{-}^{A}\right) \\
& o_{A^{\prime}} \chi_{+}^{A^{\prime}}-o_{A^{\prime}} \chi_{-}^{A^{\prime}}=-i e \tilde{f}\left(A o_{A^{\prime}} \chi_{+}^{A^{\prime}}+(1-A) o_{A^{\prime}} \chi_{-}^{A^{\prime}}\right)
\end{aligned}
$$

Once again we encounter the "ambiguity" $A$ arising from the product of $\delta$ with $\theta_{+}$. As has been our strategy in the previous paragraphs we invoke the conservation law of the Dirac-current

$$
\partial_{a} J^{a}=0 \quad J^{a}=\psi^{A} \bar{\psi}^{A^{\prime}}+\chi^{A^{\prime}} \bar{\chi}^{A}
$$

which in the smooth context is a direct consequence of (24). The conservation requirement is equivalent to

$$
\begin{aligned}
& \delta(p x)\left(\left(o_{A} \psi_{+}^{A}\right)\left(o_{A^{\prime}} \bar{\psi}_{+}^{A^{\prime}}\right)-\left(o_{A} \psi_{-}^{A}\right)\left(o_{A^{\prime}} \bar{\psi}_{-}^{A^{\prime}}\right)+\right. \\
&\left.\left(o_{A} \chi_{+}^{A}\right)\left(o_{A^{\prime}} \bar{\chi}_{+}^{A^{\prime}}\right)-\left(o_{A} \chi_{-}^{A}\right)\left(o_{A^{\prime}} \bar{\chi}_{-}^{A^{\prime}}\right)\right) \approx 0
\end{aligned}
$$

Re-arranging terms and using (28) we find, not unexpectedly, $A=1 / 2$, which in turn yields for the junction conditions at $p x)=0$

$$
\begin{aligned}
o_{A} \psi_{+}^{A} & =\frac{1-i \frac{e}{2} \tilde{f}}{1+i \frac{e}{2} \tilde{f}} o_{A} \psi_{-}^{A}, \\
o_{A} \chi_{+}^{A} & =\frac{1-i \frac{e}{2} \tilde{f}}{1+i \frac{e}{2} \tilde{f}} o_{A} \chi_{-}^{A} .
\end{aligned}
$$

Note that in contrast to the Klein-Gordon particle no further constant appears. The current itself is simply associated to its classical parts above and below the pulse. In this regard the Dirac-particle is simpler than its Klein-Gordon analogue. 


\section{Conclusion}

We have considered the scattering of charged classical and quantum particles by impulsive electromagnetic waves. The problem is reduced to the matching of free solutions above and below the pulse. The theory of Colombeau generalized functions was applied to give a meaning to products of singular terms, however leading to undetermined constants. We have shown that by implementing conservation laws that follow automatically from the equation of motion for smooth solutions, allows one to determine these constants. As examples we discussed the scattering of relativistic charged point particles and their quantum analogues i.e. charged Klein-Gordon and Dirac fields. In all cases we obtained a unique scattering amplitude. A natural step further is to extend our approach to particles with vectorial charge structure as well as gravity. We think that this method can be applied to similar physical situations, whenever the scattering source can be modeled by impulsive waves. 


\section{References}

[1] Colombeau J F, Multiplication of Distributions, LNM 1532, Springer 1992

[2] DeVega H J and Sanchez N, Nucl.Phys.B 317, 731 (1989)

[3] Luosto C and Sanchez N, Nucl. Phys. B 355, 231 (1991)

[4] Aichelburg P and Sexl R, Gen. Relat. Gravit. 2: 303 (1971)

[5] Rodnianski I and Luk J, arXiv:1209.1130, (2014)

[6] Kunzinger M and Steinbauer R, J.Math.Phys. 401479 (1999)

[7] Balasin H, Class. Quantum Grav. 14455 (1997)

[8] Penrose R, Ann.Phys. 10 171, (1960) 


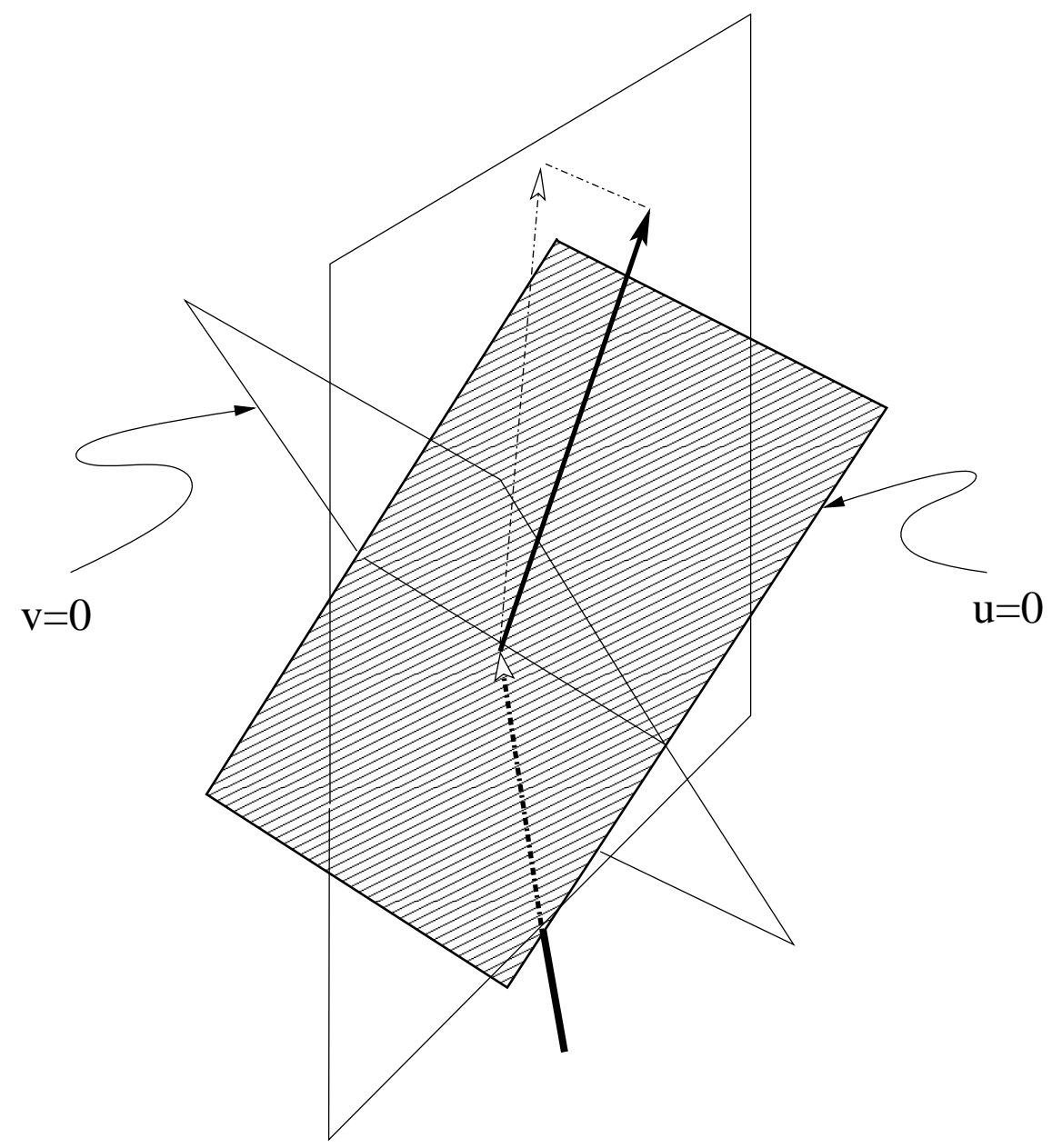

Figure 1: The figure shows, schematically, the scattering of an incident particle with $\tilde{x}^{i}=0$ by an impulsive electromagnetic field supported at $u=0$. Notice that the particle is not only scattered within its plane of incidence but also off it 